\title{
VALENCIANOS Y ARROZ EN LAS MARISMAS DEL GUADALQUIVIR
}

\author{
José GONZÁLEZ ARTEAGA *
}

\section{INTRODUCCIÓN}

Dentro del vasto territorio que son las Marismas del Guadalquivir (140.000 Has.), nos encontramos con que un buen porcentaje de ellas, concretamente el 26,5 por 100 (36.000) pertenece aún (diciembre de 1993) al término municipal de Puebla del Río. Dicho término marismeño se lo reparten tres islas como consecuencia de los diversos brazos en que se divide el Guadalquivir desde Sevilla a su desembocadura: Islas Mayor, Menor y Mínima. En cada una de ellas, y, a partir de la segunda década del siglo actual, han ido apareciendo, gracias fundamentalmente a la iniciativa privada, una serie de poblados (véanse planos adjuntos) en los que los valencianos jugaron un papel primordial ${ }^{1}$.

\section{LA POBLACIÓN VALENCIANA EN LAS MARISMAS}

$\mathrm{Al}$ acometer el estudio de la población marismeña, lo primero que tenemos que constatar es el avance que se produjo en una parte sustancial de ellas -en Ias "islas"- desde el momento que hemos escogido para iniciar el trabajo (1925) hasta el comienzo de los años ochenta en que se estabilizó. Como se puede

1. Las fuentes a las que hemos acudido para establecer la evolución de dichos poblados y de sus gentes han sido unos padrones hallados en el Archivo Municipal de Puebla del Río pertenecientes a los años 1925, 1935, 1970 y 1981; los Censos de los Sidicatos Arroceros de Sevilla, Villafranco y Las Cabezas de San Juan; y, sobre todo, las encuestas realizadas a los primeros valencianos que hicieron acto de presencia por estas tierras. También, y para recomponer la dinamicidad de dicha población, acudimos a los Libros Parroquiales y al Juzgado de la citada localidad, con eI fin de obtener datos de nacimientos y óbitos y, con ello, dar forma a lo sustancial del trabajo.

* Doctor en Historia. 
observar en el cuadro 1 y en el gráfico 1, en los primeros años de la serie (1925 y 1935) no existe aún ni un sólo valenciano, lo cual es fácil de explicar: en 1925 no aparecían todavía los poblados, y las marismas se presentan como un gran erial donde la presencia del hombre destacaba por su ausencia; mientras que en 1935, aunque ya existían, habían sido obra de una compañía extranjera que en plan experimental quiso poner en cultivo estas tieras, pero con unas perspectivas tan limitadas y especulativas que no tuvieron ni la continuidad ni la expansión necesaria como para que gentes de otros lugares vinieran en busca de oportunidades, limitándose sólo a dar trabajo a jornaleros de los pueblos limítrofes, que una vez terminada la jornada de trabajo volvían a sus casas. En 1955 el panorama cambia por completo, y al establecerse el arroz en las marismas de una forma seria y planificada a partir de 1937, comienzan a llegar, a partir de los años cuarenta, los primeros valencianos y de otros lugares de la geografía española (Anexo 1); primero de una forma tímida, despúes algo más masivamente. Ello hace que en esta última fecha (1955) aparezcan ya 270 valencianos establecidos en los diferentes poblados, lo que suponía el 7,2 por 100 de la población total de estos lugares. Pero es 1970, al compás del "boom" del arroz (que se produce en la década de los sesenta), cuando se aprecia su espectacular llegada, doblándose en número, aunque en porcentajes sólo suponga un punto, debido a que también se ha multiplicado por dos la población total de dichos poblados. Una vez pasados los momentos de euforia, sólo quedan en la zona aquéllos que han logrado hacerse con un puñado de tierras que le permiten una cierta estabilidad; el resto vuelve a sus lugares de origen o bien se establecen en los pueblos próximos o en la capital -Sevilla- si han sido capaces de reunir una aceptable fortuna, ya que las marismas no reúnen condiciones aceptables de habitabilidad. Aún así, representan, en 1981 , el 6,7 por 100 de las gentes marismeñas.

En este rápido balance constatamos que, a pesar de lo que se ha venido manteniendo, la presencia de valencianos en las "islas del Guadalquivir" no ha sido tan masiva como se creía, pero lo que sí es cierto es que fueron los elementos más atractvos, y en su poder cayeron una buena parte de las tierras puestas en cultivo. Hay que hablar, pues, más de calidad que de cantidad cuando se alude a la presencia de valencianos en las marismas. Con todo, hay que tener también e cuenta que a ellos se sumaron bastantes murcianos, catalanes, y almerienses que, por estar en contacto con las tierras de la Albufera, pusieron su granito de arena en el desarrollo del arrozal sevillano.

Si nos ocupamos ahora de la estructura de la población por sexos y grupos de edad, debemos hacer constar, en primer lugar, que las "Marismas del Guadalquivir" han sido tradicionalmente un lugar de inmigración adonde llegaban y se establecían hombres para guardar el ganado localizado en los "hatos" que desde 


\section{NUMERO DE VALENCIANOS EN LAS MARISMAS}

\begin{tabular}{|c|c|c|c|c|c|c|c|c|c|c|c|c|c|c|c|c|c|c|c|c|c|c|c|}
\hline \multirow[t]{3}{*}{ Años } & \multicolumn{10}{|c|}{ Isla Mayor } & \multicolumn{8}{|c|}{ Isla Menor } & \multirow{2}{*}{\multicolumn{2}{|c|}{ J }} & & & \\
\hline & \multicolumn{2}{|c|}{ A } & \multicolumn{2}{|c|}{ B } & \multicolumn{2}{|c|}{$\mathrm{C}$} & \multicolumn{2}{|c|}{ D } & \multicolumn{2}{|c|}{ E } & \multicolumn{2}{|c|}{$\mathrm{F}$} & \multicolumn{2}{|c|}{$\mathrm{G}$} & \multicolumn{2}{|c|}{$\mathrm{H}$} & \multicolumn{2}{|c|}{ I } & & & \multicolumn{2}{|c|}{ K } & Totales \\
\hline & $\mathrm{T}$ & $\%$ & $\mathrm{~T}$ & $\%$ & $\mathrm{~T}$ & $\%$ & $\mathrm{~T}$ & $\%$ & $\mathrm{~T}$ & $\%$ & $\mathrm{~T}$ & $\%$ & $\mathrm{~T}$ & $\%$ & $\mathrm{~T}$ & $\%$ & $\mathrm{~T}$ & $\%$ & $\mathrm{~T}$ & & $\mathrm{~T}$ & $\%$ & $\mathrm{~T} \%$ \\
\hline 1925 & - & - & - & - & - & - & - & - & - & - & - & - & - & - & - & - & - & - & - & - & - & - & - \\
\hline $1935(1)$ & - & - & - & - & - & - & - & - & - & - & - & - & - & - & - & - & - & - & - & - & - & - & - \\
\hline 1955 & 178 & 4,7 & 31 & 0,8 & 25 & 0,6 & 6 & 0,1 & - & - & - & - & - & - & - & - & 19 & 0,5 & 11 & 0,3 & - & - & $2707,2(2)$ \\
\hline 1970 & 515 & 7,4 & 2 & 0,2 & - & - & 12 & 0,1 & 10 & 0,01 & 13 & 0,1 & 9 & 0,01 & - & - & - & - & 5 & 0,07 & - & - & 5668,1 \\
\hline 1981 & 387 & 6,6 & 4 & 0,06 & - & - & - & - & 4 & 0,06 & - & - & - & - & - & - & - & - & - & - & - & - & $395 \quad 6,7$ \\
\hline
\end{tabular}
A. Villafranco.
B. Alfonso XIII.
C. Ricón de los Lirios.
D. Queipo de Llano.
E. Diseminados Isla Mayor.
F. Cotos Regables.
G. S. Vicente Ferrer.
H. La Compañía.
I. Diseminados Isla Menor.
J. Isla Mínima.
K. Colinas.

Notas: 1. Aparecen ya algunos valencianos y levantinos. 2. \% hallados sobre el total de la población de los poblados. En este año era de 3.734; en 1970 de 6.943 y en 1981 de 5.839 . 


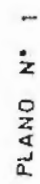

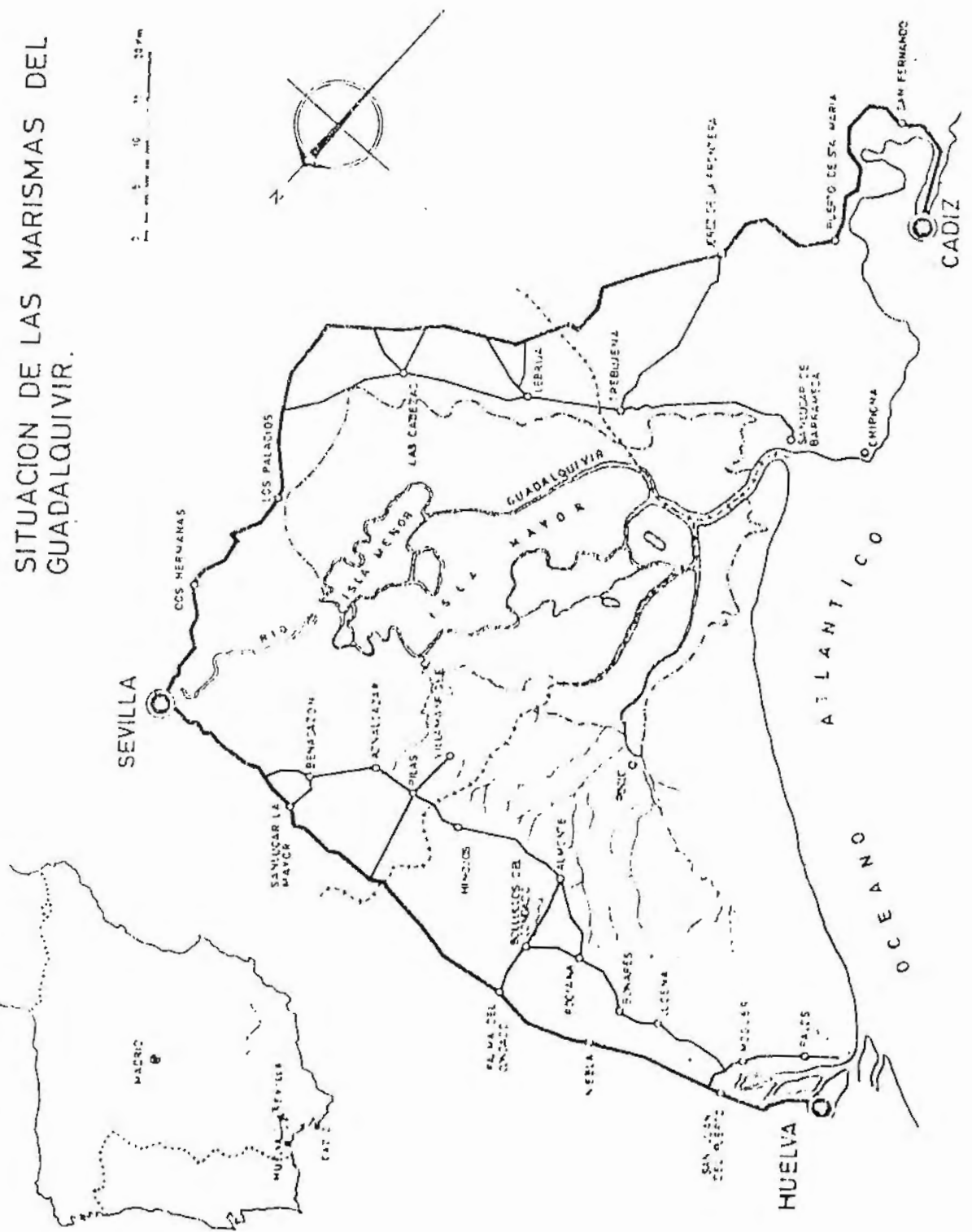




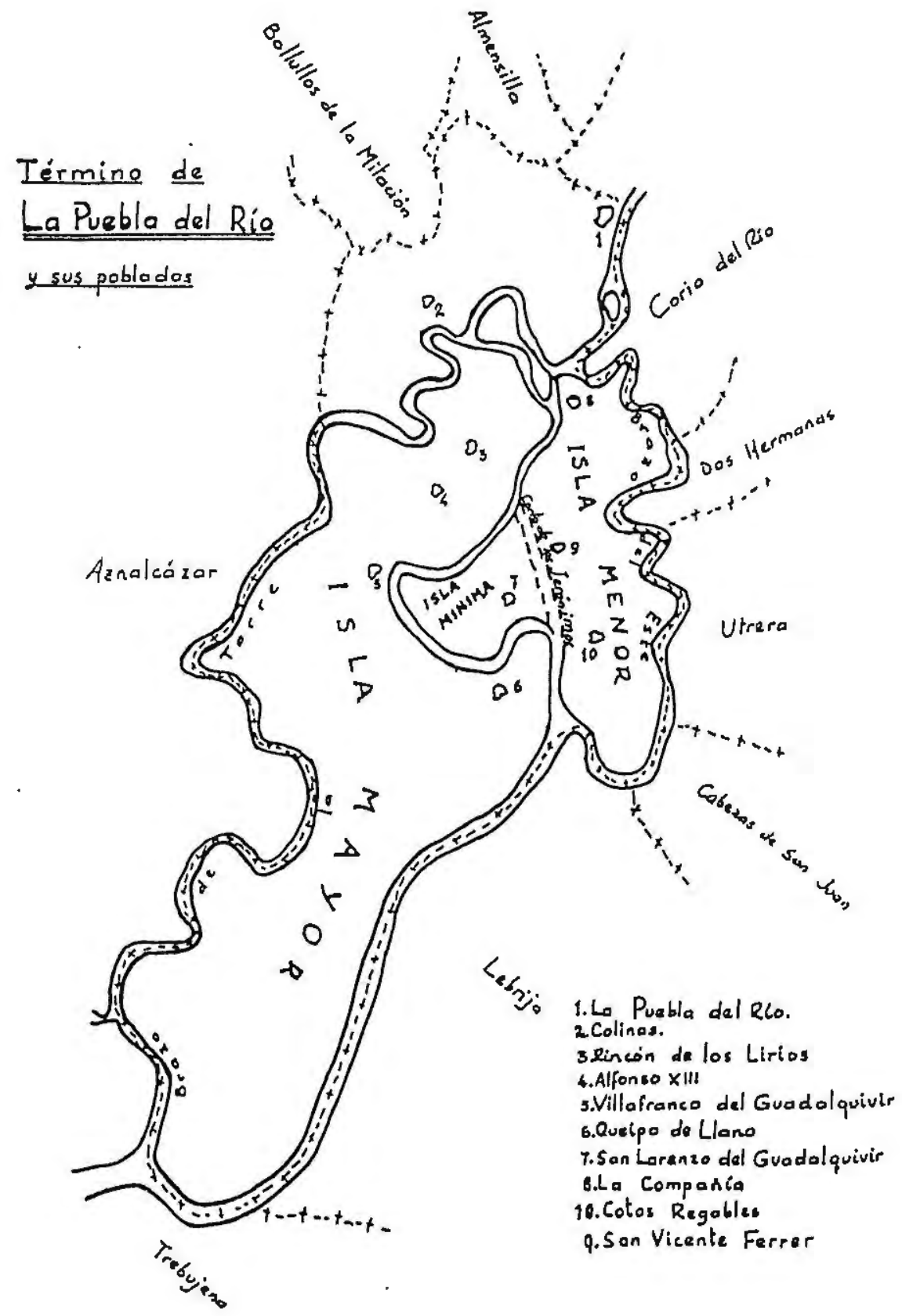


antiguo habían ido apareciendo por la gran planicie. Normalmente eran, pues, hombres solos los que aparecían desperdigados por esos desolados parajes, no siendo hasta 1935 -momento en el que las "islas" empiezan a ponerse en explotación-cuando comienzan a llegar con sus familias, lo que hace que las diferencias entre varones y hembras se amortigüe, como pone de manifiesto el cuadro 2 para el año 1955 donde prácticamente las cifras entre ambos sexos están igualadas.

Lo anterior queda también patente en los cuadros siguientes. En el 3 se observa que el grueso mayor se concentra en el grupo de los que tenían entre 16 y 65 años, presentando ya los niños una cifra nada despreciable, mientras que los mayores cuentan menos. El fenómeno anterior puede hacerse extensivo a la población del total de todos los núcleos marismeños, como se apunta en nota 1 de ese mismo cuadro. La misma conclusión es observable en el análisis del cuadro 4 , donde es ostensible la presencia de las mujeres valencianas ( $\mathrm{s} / \mathrm{l}$ ) que han llegado a la Isla acompañando a sus maridos (jornaleros y agricultores) para dedicarse, como un bracero más, a las labores agrícolas, repartiéndose aquellos el mismo porcentaje; los escolares alcanzan igualmente una cifra apreciable.

Todo ello nos hace pensar y nos ratifica que, a diferencia de lo que ocurría en épocas pasadas con gentes de otros lugares, los valencianos llegan a estas tierras con sus familias y con la idea fija de no marcharse de inmediato o nunca; de ahí que hayan sido los primeros que llegaron y los que más raíces han echado (Anexo 1).

Esto último se hace igualmente ostensible en el cuadro 5 en el que al ver los años de residencia de los valencianos, se observa que todos llegaron entre 1937 y 1955 , o sea en los años en que comienza a ponerse en cultivo el arroz.

\section{CUADRO II \\ DISTRIBUCIÓN DE LA POBLACIÓN VALENCIANA EN LAS MARISMAS POR SEXO, 1955}

Año Sexo Total población valenciana en las Marismas
\% sobre la población de los poblados

\begin{tabular}{llll}
\hline 1955 & V & 142 & 3,8 \\
& $H$ & 122 & 3,2
\end{tabular}

Totales

264

7,0 


\section{CUADRO III \\ DISTRIBUCIÓN DE LA POBLACIÓN VALENCIANA EN LAS MARISMAS POR GRUPOS DE EDAD. 1955}

\begin{tabular}{rrrc} 
Año & Grupos de edad & Totales & $\begin{array}{c}\text { \% Sobre la población } \\
\text { de los poblados 1 }\end{array}$ \\
\hline 1955 & $0-15$ & 73 & 5,1 \\
& $16-65$ & 188 & 8,3 \\
& +65 & 3 & 5,3
\end{tabular}

FUENTE: Padrón de 1955.

ELABORACIÓN: Propia

\section{CUADRO IV \\ DISTRIBUCIÓN DE LA POBLACIÓN VALENCIANA EN LAS MARISMAS POR OCUPACION. 1955}

\begin{tabular}{llcc} 
Año & Ocupación & Cifras absolutas & Cifras relativas \\
\hline 1955 & Jornaleros & 47 & 17,8 \\
& Agricultores & 46 & 17,4 \\
& S/L & 90 & 34,1 \\
& Escolares & 71 & 26,9 \\
& Otras & 10 & 3,7 \\
\hline Totales & & 264 & 100,0
\end{tabular}

FUENTE: Padrón de 1955.

ELABORACIÓN: Propia

\section{CUADRO V}

\section{AÑOS DE RESIDENCIA EN LAS ISLAS DE LOS VALENCIANOS. 1955}

\begin{tabular}{lccc} 
Año & Tiempo de residencia (2) & Cifras absolutas & $\%$ \\
\hline $1955(1)$ & $0-10$ & 238 & 90,1 \\
& $11-18$ & 15 & 5,6 \\
& +18 & 4 & 1,5
\end{tabular}

Totales

257

FUENTE: Padrón de 1955.

ELABORACIÓN: Propia

NOTAS: 1 . Hemos hecho esta distribución para saber los que vinieron atraídos por el arroz (0-18), o sea entre 1937 y 1955, que son los años en que se comienza a poner en cultivo este cereal en las marismas. 2. Prácticamente se desplazan familias enteras y, en muchos casos, parejas de recién casados que tienen sus hijos ya en estas tieras aunque los hayamos contabilizado como valencianos, en la categoría de "escolares". 3. Los siete que faltan no constan este concepto. 

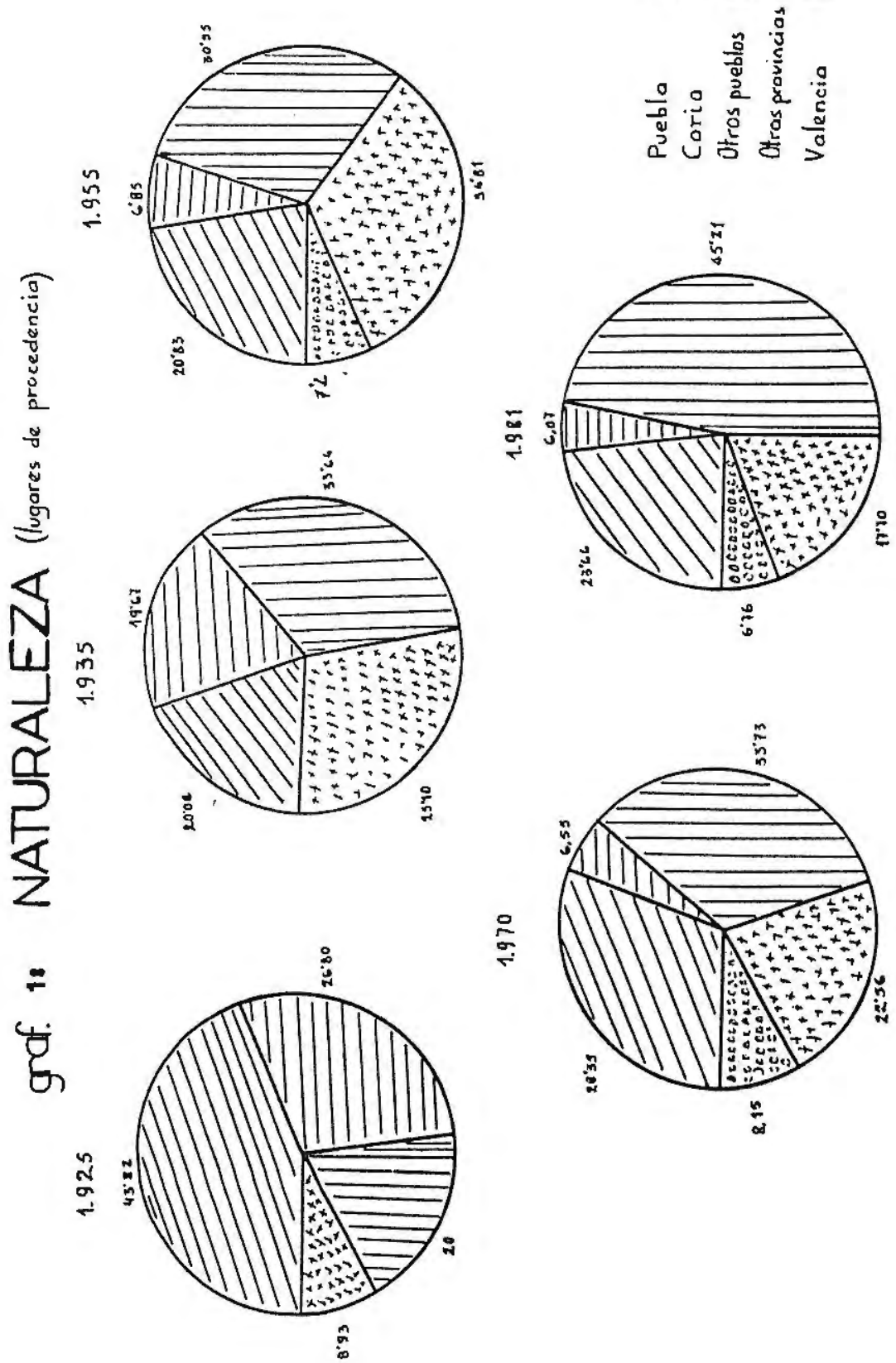
Visto la manera en que van llegando los valencianos, ocupémonos ahora de cómo comienzan a marcharse a partir de 1970. Vamos a tratarlo de una manera global, haciendo especial hincapié en el caso de los levantinos, pues consideramos que se sale de la norma general. Así, hasta 1955 y durante toda la década de los sesenta, la Isla no deja de ganar población, como hemos tenido ocasión de exponer, y prácticamente no se producen bajas por emigración, hasta el punto que en los padrones de 1925, 1935 y 1955 no se recogen; no sucede lo mismo a partir de 1970 en donde se anotan 909 bajas, cifra que se nos antoja bastante alta. De ese cerca del millar de emigrantes 447 (casi la mitad) salieron de Villafranco, repartiéndose el resto como muestra el cuadro 6.

Al detenernos en analizar el tipo de emigración que se dio, observamos que en este año (1970) salieron 169 familias enteras, lo que supone el 10,47 por 100 del total de las familias. Por otro lado, su composición arrojaba una media de 4,90 individuos/familia, lo que hacía un total de 726,70 personas las que salieron en bloques familiares, mientras que el resto (182) lo hicieron aisladamente. Se impone, pues, la emigración en bloques familiares, lo cual, creemos, dice mucho sobre el estancamiento poblacional de la Isla por estas fechas.

El primer lugar, en pérdidas de familias lo ocupa, como era de esperar, Villafranco, seguido de Alfonso XIII, Queipo de Llano y S. Vicente Ferrer; los demás arrojan cifras menos importantes, pero de todos salen algunas.

En cuanto a los lugares hacia donde se dirigen, sobresale Barcelona y su provincia, y si a ello les sumamos los que marchan a Gerona y Tarragona, se constata que más del 40 por 100 lo hacen a Cataluña. El segundo lugar lo ocupan los que salen para otros puntos de la provincia sevillana, como es el caso de los valencianos, que al haberse afincado, aclimatado y hecho fortuna dejan los poblados para pasar a engrosar los padrones de Puebla del Río, Coria del Río y Sevilla; otros, por último, se deciden a volver a Valencia.

Pero ¿por qué se marchan? El motivo es vario: por un lado están los que se sienten desengañados desde el primer momento y vueIven a su tierra; en segundo lugar, aquéllos otros que no habiendo alcanzado el "maná" esperado en las tierras marismeñas se desplazan a otros lugares en busca de fortuna; y en tercer lugar, la mecanización que se produce a partir de mediados de los sesenta y se incrementa enormemente en los setenta. Como consecuencia, los que no habían reunido un mínimo suficiente de hectáreas para poder subsistir y tenían que trabajar por cuenta ajena a terceros -ya que de otra manera no podían salir adelante, al reducirse la mano de obra tan drásticamente- no tuvieron más remedio que marcharse. Pero, insistimos, los que menos abandonaron fueron los valencianos.

En general, ese descontento al que estamos aludiendo se acrecienta a medidas que pasan los años, pues se puede verificar que si en 1971 salieron 147 indivi- 
CUADRO VI

BAJAS. PADRON DE 1970

\begin{tabular}{|c|c|c|c|c|c|c|c|c|c|c|c|c|c|c|c|c|c|c|c|c|}
\hline \multirow[b]{3}{*}{ Tolales } & \multicolumn{8}{|c|}{ Isla Mayor } & \multicolumn{8}{|c|}{ Isla Menor } & & & \multicolumn{2}{|c|}{ totales } \\
\hline & \multicolumn{2}{|c|}{ A } & \multicolumn{2}{|c|}{$\mathrm{B}$} & \multicolumn{2}{|c|}{$\mathrm{C}$} & \multicolumn{2}{|c|}{$\mathrm{D}$} & \multicolumn{2}{|c|}{$\mathrm{F}$} & \multicolumn{2}{|c|}{ G } & \multicolumn{2}{|c|}{$\mathrm{H}$} & \multicolumn{2}{|c|}{$\mathrm{I}$} & \multicolumn{2}{|c|}{$\mathrm{K}$} & \multirow{2}{*}{$\begin{array}{c}\text { C.Abs. } \\
909\end{array}$} & \multirow{2}{*}{\begin{tabular}{|c|}
$\%$ \\
$13,09(7)$ \\
\end{tabular}} \\
\hline & 447 & 49,17 & 169 & 18,59 & 5 & 0,55 & 144 & $15,84(2)$ & 31 & 3,41 & 69 & 7,59 & 13 & 1,43 & 23 & 2,53 & 8 & 0,88 & & \\
\hline Familias enteras & 90 & 53,25 & 29 & 17,15 & 1 & 0,59 & 25 & 14,79 & 6 & 3,55 & $11(3)$ & 6,50 & 2 & 1,18 & $4(6)$ & 2,36 & 1 & 0,59 & 169 & $10,47\left(^{*}\right)$ \\
\hline $\begin{array}{l}\text { LUGARES DE NMIGRACIÓN } \\
\text { BARCELONA Y SU PROVINCIA }\end{array}$ & 166 & 18,26 & 97 & 10,67 & - & - & 61 & 6,71 & 5 & 0,55 & $\rightarrow$ & - & - & - & - & - & 1 & 0,11 & 330 & 36,30 \\
\hline VALENCIA Y SU PROVINCIA (1) & 83 & 9,13 & - & - & - & - & 21 & 2,31 & - & - & - & - & - & - & - & - & - & - & 104 & $11,44(9)$ \\
\hline SEVILLA Y SU PROVINCIA & 92 & 10,12 & 36 & 3,96 & 5 & 0.55 & 27 & 2,97 & 24 & 2,64 & 48 & 5,28 & 13(5) & 11,43 & 23 & 2,53 & 7 & 0,77 & 275 & 30.25 \\
\hline GERONA Y TARRAGONA & 19 & 2.09 & 13 & 1.43 & - & - & 5 & 0,55 & - & - & $3(4)$ & 0,33 & - & - & - & - & - & - & 40 & 4,40 \\
\hline OTRAS PROVINCIAS & 87 & 9,57 & 23 & 2,53 & - & - & 30 & 3,30 & 2 & 0,22 & 18 & 1,98 & - & - & - & - & - & - & 160 & 17,60 \\
\hline Años en que inmigran & & & & & & & & & & & & & & & & & & & & \\
\hline 1971 & 56 & 6,16 & 35 & 3,85 & - & - & 43 & 4,73 & 5 & 0,55 & 4 & 0,44 & - & - & 4 & 0,44 & - & - & 147 & 16,17 \\
\hline 1972 & 44 & 4,84 & 24 & 2,64 & - & - & 28 & 3,08 & 3 & 0,33 & 20 & 2,20 & - & - & 8 & 0,88 & l & 0,11 & 128 & 14,08 \\
\hline 1973 & 108 & 14,88 & 47 & 5,17 & 1 & 0,11 & 24 & 2,64 & 3 & 0,33 & 30 & 3,30 & 6 & 0,66 & - & - & - & - & 219 & 24,09 \\
\hline 1974 & 133 & 14,63 & 34 & 3,74 & - & - & 21 & 2,31 & 12 & 1,32 & 8 & 0,88 & - & - & - & - & - & - & 208 & 22,88 \\
\hline 1975 & 106 & $\$ 1,66$ & 29 & 3,19 & 4 & 0,44 & 28 & 3,08 & 8 & 0,88 & 7 & 0,77 & 7 & 0,77 & 11 & 1,21 & 7 & 0,77 & 207 & 22,77 \\
\hline
\end{tabular}

\section{NOTAS:}

(1) La mayoría se desplaza a Coria.

(2) Se incluyen también los diseminados de Queipo.

(3) Las 11 familias suponen la tortalidad: 69.

(4) En este caso es a Lérida.

(5) De las 13,10 lo hacen a Coria.
(6) De nuevo esas cuatro familias suponen la totalidad: 23.

(7) Ese \% se ha hallado sobre el total de la población: 6.943 .

(8) Hallado sobre el total de las familias de ese año: 1.613.

(9) La mayoría son gentes que vuelven a su tierra. 
duos, cuatro años más tarde, en 1975 , lo hicieron ya 207. De todas maneras, esa tendencia a salir de la Isla se va deteniendo progresivamente, llegándose a 1981 (cuadro 7) en que sólo lo hacen 206 (el 3,52 por 100 de la población total de los núcleos). La inmensa mayoría son de nuevo de Villafranco, y en menor medida de Alfonso XIII y S. Vicente Ferrer. Por otro lado, se nota que el número de familias que salen completas han bajado sensiblemente.

En este padrón no se recogen los lugares hacia donde se dirigen, sino solamente si lo hacen por "cambio de domicilio" (o sea de manera definitiva) o "por trabajo" (transitoriamente). Dominan los primeros (el 84,95 por 100), lo que demuestra que la lsla ha llegado al límite de su "boom" y lo único que le espera es el estancamiento o la marcha atrás.

Esto queda también evidente si nos fijamos en que, aunque menos que en los años sesenta, también a medida que avanza en el tiempo la emigración aumenta, si bien nos encontramos con un año que destaca sobre los demás, 1983, con 141, siendo sintomático que coincida con la gran sequía que asoló nuestra región y que hizo que no se cultivase arroz. Es obvio, pues, hacer referencia a la interrelación existente entre población y cultivo arrocero.

No podemos acabar este apartado sin hacer alusión de la idiosincrasia del hombre marismeño y de cómo la llegada de gentes foráneas (entre los que se encuentran, en un primer plano, los valencianos) le han cambiado por completo. Efectivamente, en ese ya antiguo hombre marismeño se encontraban todos los atributos que definían al campesino de andalucía Ia Baja: la gracia, la sobriedad, con un cierto color de estoicismo, la indiferencia escéptica ante los negocios públicos, la pasión desbordada ante los conflictos personales, el analfabetismo sabio y profundo que se traducía en sentencias -piénsese en la figura literaria de "El Séneca"- y rehuía el tópico; sus costumbres, triviales y generosas, eran restos indelebles de una civilización superior que el análisis más superficial en el espíritu de estos andaluces descubría.

Todo ello adobado con lo que la propia tierra aportaba; y esta tierra hizo al hombre marismeño, antes que nada, pastor y cazador, oficios ambos que le dieron un carácter independiente e introvertido; y es caballista, lo que es sinónimo de arrogante y orgulloso; y rociero, que conlleva devoción y apasionamiento.

Ese es el cliché del antiguo hombre marismeño. Pero ese hombre prácticamente ha desaparecido, y, en su lugar, ha surgido otro nuevo, con mentalidades y trabajos distintos; son el agricultor y el pescador; hombres que vinieron, muchos de ellos de fuera, que comenzaron a interpretar de manera distinta a las marismas, que sufrieron en ellas, que se apegaron a ellas y que han asumido mucho de su cultura y folcklore. En conclusión, es el nuevo hombre marismeño, más pragmático, realista e inquieto; pero son nuevos tiempos y a ello se imponen nuevas actitudes. 
CUADRO VII

BAJAS. PADRON DE 1981

\begin{tabular}{|c|c|c|c|c|c|c|c|c|c|c|c|c|c|c|c|c|}
\hline & \multicolumn{4}{|c|}{ Isla Mayor } & \multicolumn{8}{|c|}{ Isla Menor } & & & \multicolumn{2}{|c|}{ Totales } \\
\hline & \multicolumn{2}{|c|}{ A } & \multicolumn{2}{|c|}{ B } & \multicolumn{3}{|c|}{$F$} & \multicolumn{2}{|c|}{ G } & \multicolumn{2}{|c|}{$\mathrm{H}$} & I & \multicolumn{2}{|c|}{$\mathrm{K}$} & & \\
\hline & C. Abs. & $\%$ & C. Abs. & $\%$ & C. Abs. & $\%$ & C. Abs. & $\%$ & C. Abs. & $\%$ & C. Abs. & $\%$ & C. Abs. & $\%$ & C. Abs. & $\%$ \\
\hline Totales & 138 & 66,99 & 27 & 13,10 & 8 & 3,83 & 22 & 10,67 & 5 & 2,42 & 1 & 0,48 & 5 & 2,42 & 206 & $3,52(1)$ \\
\hline Familias enteras & 23 & 65,71 & 5 & 14,28 & 2 & 5,71 & 3 & 8,57 & 1 & 2,85 & - & - & 1 & 2,85 & 35 & $2,52(2)$ \\
\hline Causas de las Bajas & & & & & & & & & & & & & & & & \\
\hline Cambio de domicilio & 115 & 55,82 & 23 & 11,16 & 8 & 3,88 & 22 & 10,67 & 1 & 0,48 & 1 & 0,48 & 5 & 2,42 & 175 & 84,95 \\
\hline Trabajo & 24 & 11,65 & 7 & 3,39 & - & - & - & - & - & - & - & - & - & - & 31 & 15,04 \\
\hline Años en que emigran & & & & & & & & & & & & & & & & \\
\hline En 1981 & - & - & 7 & 3,39 & 4 & 1,94 & - & - & - & - & - & - & - & - & 11 & 5,33 \\
\hline En 1982 & 17 & 8,25 & 1 & 0,48 & 4 & 1,45 & 5 & 2,42 & - & - & - & - & - & - & 26 & 12,62 \\
\hline En 1983 & 99 & 48,05 & 19 & 9,22 & - & - & 17 & 8,25 & 5 & 2,42 & 1 & 0,48 & - & - & 141 & 68,44 \\
\hline En 1984 & 22 & 10,67 & - & - & 1 & 0,48 & - & - & - & - & - & - & 5 & 2,42 & 28 & 13,59 \\
\hline
\end{tabular}

(1) \% sobre el total de población: 5.839.

(2) \% sobre el total de las familias: 1.385 . 


\section{IMPRESIONES, MEDIO NATURAL Y PROBLEMÁTICA A LA QUE SE ENFRENTARON LOS PRIMEROS VALENCIANOS QUE LLEGAN A LAS ISLAS}

La impresión que les causaron las marismas a los primeros valencianos que llegaron a principios de los años cuarenta fue pésimo, lo que no debe sorprendernos, ya que se encontraron con unos terrenos completamente baldíos y vírgenes, inmensos y en donde dominaba, por una parte, la vegetación rala aprovechada sólo por el ganado, y, por otra, el paludismo, un mal endémico en nuestras marismas, que, aunque no les era desconocido, aquí atacaba con una virulencia inusitada. El medio, pues, no podía ser más hostil, y ello les imbuyó una idea de impotencia que a muchos les hizo desistir del empeño y volver; sólo los más fuertes, avispados o con un golpe de fortuna se quedaron e hicieron fortuna.

Pero ¿cómo y por qué vienen los valencianos? En un primer momento se desplazan solos para ver las perspectivas que ofrecen las nuevas tierras, y una vez establecidos vuelven por sus familias si las tienen, y sin no la forman y regresan con ella, ya que se dan cuenta de las dificultades que presenta el estar aislado en ese medio. En el supuesto de que no observaran unas posibilidades halagüeñas no lo hacen, quedándose en sus lugares.

Respecto a la clase social a la que pertenecían los valencianos que acuden a la Isla Mayor nos encontramos de todo. Unos -los más modestos- fueron campesinos sin otro patrimonio que sus brazos y sin nada que perder con su venida y con mucho que ganar, como sucedió en bastantes casos. Otros tenían algunas tierras allí, pero con gran afán por mejorar su situación las vendieron e invirtieron aquí. $\mathrm{Y}$, finalmente, gentes algo acomodadas que vinieron con la intención de incrementar su patrimonio y con sus ganancias invertir de nuevo en Valencia en naranjales o arrozales. Unos lo hicieron así, mientras que la mayoría vieron el gran futuro que ofrecían y se quedaron. En muchos casos, el componente de una familia llega, y si con el paso del tiempo le ha ido bien, tira del resto, lo que hace que nos encontremos en los censos arroceros con verdaderas "sagas" familiares; de ahí que los apellidos se repitan con bastante frecuencia. Estas gentes acomodadas, a las que podríamos denominar commo "capitalistas", vienen muchas veces con hombres de su cofianza (capataces), que con el tiempo se independizan y llegan a formar unas medianas o grandes fortunas, dependiendo de la capacidad de trabajo, inteligencia y adaptación al medio, tanto al físico como al humano.

Muchos -sobre todo entre los acomodados- comenzaron como arrendatarios, haciéndose la renta por contrario simple de seis años, con un canon muy bajo; sin embargo, esto duró muy poco y sólo fue el anzuelo que utilizó la Compañía Beca para atraerse a las gentes. En esta modalidad, la Compañía lo ponía todo (abono, 
semillas, agua, etc.) a cambio de quedarse con el 25 por 100 de la producción. Más tarde se comenzó a comprar, y cada uno se hizo con lo que podía según sus circunstancias económicas, con planos por delante y escogiendo lo que quería y podía. En 1946 la hectárea se podía adquirir por 16.000 pesetas, con un interés pequeño (del 3 ó 4 por 100).

La situación laboral con que se encuentran fue también sorprendente, ya que por aquellas fecha la jomada laboral en Valencia era de 5 horas, mientras que en Andalucía aún estaba en 8 cuando se trabajaba a jomal, y al llegar la época de plantación y siega se imponía el destajo; era el momento culminante de la explotación del hombre por el hombre, como en plena etapa caciquil. Los trabajos más duros eran la plantación, la siega, y, sobre todo, el abonado; este último se hacía a pie, con espuertas a hombros y agua hasta las rodillas; mientras que el más suave lo suponía la escarda. En 1944 un simple jomalero ganaba de 11 a 12 pesetas las 8 horas de trabajo y el manijero subía hasta las 18 ó 19. Con el destajo se incrementaba el jornal bastante, pero a cambio de un trabajo despiadado. Dichos salarios eran más altos que en todo el resto de Andalucía, pero también mucho más duro; subían anualmente entre un 5 ó 6 por 100 , descontándoseles 1,50 por comida. Si a ello le sumamos otros gastos, como cama, tabaco, vino, etc., un jornalero ahorrativo, eventual y que había llegado solo, podía ahorrar 8 ó 9 pesetas diarias. Cuando llegaba con la familia entera -que era el caso general entre los valencianos- se hacían la comida y no tenían que pagar hospedaje, lo que suponía un ahorro mayor. Ese espíritu de trabajo, economizador y sacrificado, que por sus características rayaba en lo infrahumano, fue lo que hizo posible su triunfo sobre el medio tan hostil que antes citábamos y su doblegamiento hasta conseguir hacerlo rendir, gracias a lo cual hoy pueden vivir con un cierto desahogo.

Por la esquematización de la situación que acabamos de hacer, es obvio figurarse las condiciones de las primeras viviendas: los que le trabajaban a la Compañía Beca lo hacían en barracones inmundos y se les daba la comida -un rancho horroroso-; los colonos, por su parte, vivieron primero en chozas - una para cada familia-, y, más tarde, en almacenes, separándose a las familias con una especie de tabique hecho a base de sacos, lo que no permitía ningún tipo de intimidad. Cuando pudieron, dichos colonos se fueron haciendo sus casas, sin agua, luz... o sea sin un sólo servicio. El agua estaba racionada para los empleados de la Compañía -para los que era gratuita-, mientras que el resto de los colonos y jornaleros se las arreglaban como podían, siendo lo más habitual cogerla de los canales y filtrarla de manera muy rudimentaria a base de paños. Ello era la razón de que todo habitante de los arrozales fuera un palúdico crónico, y era preceptivo a la hora de la comida tomar quinina, a pesar de lo cual las fiebres se presentaban intermitentes. Esta situación se mantuvo hasta 1946 en que se construyó un 
depósito en Alfonso XIII donde se vendía agua traída de Colinas (otro de los poblados). A partir de entonces el obrero comenzó a beber agua potable, lo que no conllevó la desaparición del paludismo, que aún en los años sesenta atacaba con fuerza.

Todo ello era impuesto y organizado por la Compañía Beca, que con sus colonos, sin embargo, llegó, en muchos casos, a un buen entendimiento, y su mentor, D. Rafael Beca, tuvo muchos rasgos humanitarios, según manifestaciones proporcionadas por los propios interesados. Según éstos, mucho más explotados se sintieron los braceros con los capataces de la propia Compañía -con los que estableció D. Rafael una especie de clientelismo o trato de favor-, que, con una cierta autonomía consentida, llegaron a montar cantinas en las que, con unos precios abusivos, esquilmaban los bolsillos de los jomaleros. Por el contrario, fueron los colonos quienes mejor trataron a estos últimos, ya que, en muchos casos, habían pasado por la misma situación.

\section{LA PROPIEDAD VALENCIANA EN EL ARROZAL SEVILLANO}

La elaboración de este apartado ha sido posible gracias a una fuente de singular importancia e insustituible -aunque poco utilizada- para el objetivo que nos proponemos: establecer la evolución de la propiedad arrocera valenciana en las Islas del Guadalquivir. Se trata, como ya hemos apuntado, de los censos del Sindicato Arrocero de Sevilla que se encuentran en la Federación de Agricultores Arroceros de España (F.S.A.A.E.) en su Delegación Zona Sur, ubicada en Sevilla.

En un primer momento, sólo aparece en la totalidad de la zona arrocera del sur de España un sindicato, el de Sevilla; así se va a mantener hasta 1962 en que surge un segundo en Las Cabezas de San Juan, para, tres años más tarde, en 1965 , quedar configurado el mapa sinndical con el nacimiento de un tercero en Villafranco del Guadalquivir. Los tres, pues, en la provincia de Sevilla.

El primer censo del que tenemos noticias se remonta a un año no especificado de principios de la década de los cincuenta, cuando todavía existía un sólo sindicato, y que no ofrece la superficie por términos, sino que se limita a los nombres de los agricultores y a la superficie total de cada uno de ellos. Por él se sabe que ya los socios totales eran 436 y la superficie cultivada de $11.747,47$ hectáreas.

En 1955 se confecciona ya uno más completo -de ahí que sea del que vamos a partir-, en donde aparece, junto a los apellidos y nommbres por orden alfabético, el número de orden, el término municipal en que se halla la explotación y la superficie, junto al canon que pagaba el agricultor por hectárea, que era, concretamente, de 84 pesetas (es el único donde aparece este concepto). 
No es hasta la campaña 1965-66 cuando quedan constituidos los tres sindicatos y cuando, por primera vez, aparecen desglosados. En dicho censo se especifican los nombres, superficies y domicilio del cultivador, no contando en el de Sevilla, donde están ubicadas las tierras, lo que sí hacen los de Las Cabezas y Villafranco.

Los censos se sucede con las mismas características hasta la campaña 1984-85 en que se constata un giro en su confección, al ofrecer una mayor amplitud de datos y más perfección. Lo primero que aparece es un resumen por partidas de la superficie cultivada, con las zonas arroceras y las superficies, especificando si son llevadas en propiedad, arrendamiento o aparcería, y el total. A continuación incluye una relación agricultor por agricultor con el nombre, número de la parcela, lugar y superficie. Todo ello se hace extensivo a los tres sindicatos.

El último analizado es el de la campaña 1991-92, en el que se observan pocas variaciones con respecto al de 1975, de ahí que hayamos decidido prescindir de su análisis.

Con ese singular material se ha podido establecer la evolución de la propiedad valenciana en los arrozales de las Marismas del Guadalquivir y llegar a una serie de premisas y conclusiones. En el cuadro 8 puede apreciarse que en 1955 los valencianos suponían la mitad de los arroceros establecidos en la zona, detentando el 36 por 100 de las tierras, situación que se mantiene inalterable durante todo el período a estudio, pues si bien hasta 1965 siguen llegando valencianos, el aumento en socios y tierras se debe al reparto que comienzan a hacer de su propiedad los agricultores pioneros entre sus familiares por razones naturales (sus hijos tienen que formar sus propias familias) y fiscales.

Para abundar algo más en lo dicho, se ha establecido en el cuadro 9 cómo se reparten los valencianos en los distintos sindicatos a partir de su aparición y hasta que se produce de nuevo su fusión en los momentos actuales. De ahí que sólo hayamos analizado los dos momentos cruciales: 1965 y 1975. Es notorio, pues, que la situación cambia poco, estando la mayoría de ellos afiliados en el de Villafranco, que es donde vivían, estando las tierras distribuidas casi a partes iguales entre éste y el de Sevilla, lo que deja de manifiesto que los mayores propietarios "huyen" de las marismas buscando mejores perspectivas de habitabilidad y de servicios sociales en la capital o en los pueblos adyacentes (Coria o Puebla del Río). El sindicato de Las Cabezas se presenta nuevamente como simbólico, y si se nota un apreciable aumento de sus tierras en 1975 es como consecuencia de haberse instalado en su término alguna sociedad llevada por valencianos y dedicarse al cultivo arrocero.

Si ahora establecemos en el arrozal sevillano una pequeña propidad -de 0,1 a 10 hectáreas-, que permite solamente ir tirando a sus poseedores; una mediana - 
CUADRO VIII

EVOLUCION DE LA PROPIEDAD EN EL ARROZAL SEVILLANO (1955-1975)

\begin{tabular}{lllllllrrr}
\hline Años & \multicolumn{2}{c}{ Total Propietarios } & \multicolumn{2}{c}{ Tierras en su poder } & \multicolumn{2}{c}{ Valencianos } & \multicolumn{2}{c}{ Tierras en su poder } \\
\hline & Socios & $\%$ & Has. & $\%$ & Socios & $\%$ & Has. & $\%$ \\
\hline 1955 & 369 & 100 & $9.948,0$ & 100 & 186 & 50.4 & $3.597,5$ & 36,0 \\
1965 & 879 & 100 & $19.170,7$ & 100 & 392 & 44,4 & $7.146,2$ & 37,2 \\
1975 & 905 & 100 & $22.446,1$ & 100 & 383 & 42,3 & $8.446,6$ & 37,6 \\
\hline
\end{tabular}

FUENTE: F.S.A.A.E. Delegación Zona Sur. Sevilla

ELABORACION: Propia.

\section{CUADRO IX \\ EVOLUCION DE LA PROPIEDAD VALENCIANA POR SINDICATOS}

\begin{tabular}{cccccccccccccc}
\hline Años & \multicolumn{3}{c}{ Sto. de Villafranco } & \multicolumn{3}{c}{ Sto. de Sevilla } & \multicolumn{4}{c}{ Sto. de Las Cabezas } \\
\hline & Socios & $\%(1)$ & Has.(2) & $\%$ & Socios & $\%$ & Has. & $\%$ & Socios & $\%$ & Has. & $\%$ & \\
1965 & 260 & 66,3 & $3.480,0$ & 48,7 & 102 & 26,0 & $3.179,2$ & 44,5 & 30 & 7,6 & 487,0 & 6,8 & \\
1975 & 247 & 64,5 & $3.609,2$ & 42,7 & 101 & 26,3 & $3.806,4$ & 45,0 & 35 & 9,6 & $1.030,9$ & 12,2 & \\
\hline
\end{tabular}

NOTAS: 1 y 2: Los \% están hallados sobre el total de los arroceros valencianos y hectáreas cultivadas en las marismas por ellos. FUENTE: F.S.A.A.E. Delegación Zona Sur. Sevilla.

ELABORACION: Propia. 
CUADRO $\mathrm{X}$

ESTRUCTURA DE LA PROPIEDAD VALENCIANA EN EL AROZAL SEVILLANO (1955-1975)

\begin{tabular}{|c|c|c|c|c|c|c|c|c|c|c|c|c|}
\hline \multirow[t]{2}{*}{ Propiedad (Has.) } & \multicolumn{4}{|c|}{$1955(1)$} & \multicolumn{4}{|c|}{1965} & \multicolumn{4}{|c|}{1975} \\
\hline & Socios & $\%$ & Has. & $\%$ & Socios & $\%$ & Has. & $\%$ & Socios & $\%$ & Has. & $\%$ \\
\hline De 0,1 a 5 & 14 & 7,5 & 49,2 & 1,3 & 30 & 7,6 & 118,3 & 1,6 & 18 & 4,7 & 71,9 & 0,8 \\
\hline De 5,1 a 10 & 61 & 32,8 & 45,9 & 12,7 & 134 & 34,2 & $1.038,8$ & 14,5 & 99 & 25,8 & 799,0 & 9,4 \\
\hline De 10,1 a 20 & 72 & 38,7 & $1.051,0$ & 29,2 & 143 & 36,4 & $2.034,8$ & 28,4 & 137 & 35,7 & $1.997,8$ & 23,6 \\
\hline De 20,1 a 50 & 27 & 14,5 & 811,3 & 22,5 & 66 & 16,8 & $2.099,9$ & 29,3 & 92 & 24,0 & $2.915,0$ & 34,5 \\
\hline De 50,1 a 100 & 6 & 3,2 & 372,0 & 10,3 & 12 & 3,0 & 891,7 & 12,4 & 32 & 8,3 & $1.912,3$ & 22,6 \\
\hline Más de 100 & 6 & 3,2 & 855,0 & 23,7 & 7 & 1,7 & 962,0 & 13,4 & 5 & 1,3 & 750,4 & 8,7 \\
\hline Totales & 186 & & $3.597,5$ & & 392 & & $7.146,2$ & & 383 & & $8.446,6$ & \\
\hline
\end{tabular}

NOTAS: (1) Los \% están hallados sobre el total de propietarios valencianos y del total de sus tierras.

FUENTE: F.S.A.A.E. Delegación Zona Sur. Sevilla.

ELABORACION: Propia. 


\section{CUADRO XI}

ESTRUCTURA DE LA PROPIEDAD VALENCIANA EN EL ARROZAL SEVILLANO POR SINDICATOS. 1965

\begin{tabular}{|c|c|c|c|c|c|c|c|c|c|c|c|c|}
\hline \multirow[t]{2}{*}{ Propiedad (Has.) } & \multicolumn{4}{|c|}{ Sto. Villafranco } & \multicolumn{4}{|c|}{ Sto. Sevilla } & \multicolumn{4}{|c|}{ Sto. Las Cabezas } \\
\hline & Socios & $\%(1)$ & Has. & $\%(2)$ & Socios & $\%$ & Has. & $\%$ & Socios & $\%$ & Has. & $\%$ \\
\hline De 0,1 a 5 & 26 & 6,6 & 100,3 & 1,4 & 4 & 1,0 & 17,9 & 0,2 & - & - & - & - \\
\hline De 5,1 a 10 & 106 & 27,0 & 807,2 & 11,3 & 18 & 4,6 & 152,2 & 2,1 & 10 & 2,5 & 79,3 & 1,1 \\
\hline De 10,1 a 20 & 97 & 24,7 & $1.354,5$ & 18,9 & 32 & 8,1 & 479,9 & 6,7 & 14 & 3,5 & 200,4 & 2,8 \\
\hline De 20,1 a 50 & 26 & 6,6 & 777,2 & 10,8 & 35 & 9,0 & $1.170,5$ & 16,3 & 5 & 1,2 & 152,2 & 2,1 \\
\hline De 50,1 a 100 & 3 & 0,7 & 198,1 & 2,8 & 8 & 2,0 & 638,6 & 9,0 & 1 & 0,2 & 55,0 & 0,7 \\
\hline Más de 100 & 2 & 0,5 & 242,6 & 3,4 & 5 & 1,2 & 720,0 & 10,0 & - & - & - & - \\
\hline Totales & 260 & 66,3 & $3.480,0$ & 48,7 & 102 & 26,0 & $3.179,2$ & 44,5 & 30 & 7,6 & 487,0 & 6,8 \\
\hline $\begin{array}{l}\text { Totales de } \\
\text { arroceros y } \\
\text { hectáreas en } \\
\text { las islas }\end{array}$ & 388 & 44,2 & $5.835,4$ & 30,4 & 333 & 37,9 & $10.402,5$ & 54,3 & 158 & 17,9 & $2.932,7$ & 15,3 \\
\hline
\end{tabular}

NOTAS 1 Y 2: Los \% están hallados sobre el total de los socios valencianos y tierras en su poder en el total de los tres sindicatos.

FUENTE: F.S.A.A.E. Delegación Zona Sur. Sevilla.

ELABORACION: Propia. 


\section{CUADRO XII}

ESTRUCTURA DE LA PROPIEDAD VALENCIANA EN EL ARROZAL SEVILLANO POR SINDICATOS. 1975.

\begin{tabular}{|c|c|c|c|c|c|c|c|c|c|c|c|c|}
\hline \multirow[t]{2}{*}{ Propiedad (Has.) } & \multicolumn{5}{|c|}{ Sto. Villafranco } & \multicolumn{3}{|c|}{ Sto. Sevilla } & \multicolumn{4}{|c|}{ Sto. Las Cabezas } \\
\hline & Socios & $\%(1)$ & Has. & $\%(2)$ & Socios & $\%$ & Has. & $\%$ & Socios & $\%$ & Has. & $\%$ \\
\hline De 0,1 a 5 & 18 & 4,7 & 71,9 & - & - & - & - & - & - & - & - & - \\
\hline De 5,1 a 10 & 89 & 23,2 & 715,2 & 8,4 & 8 & 2,1 & 74,8 & 0,8 & 1 & 0,2 & 9,0 & 0,1 \\
\hline De 10,1 a 20 & 97 & 25,3 & $1.362,9$ & 16,1 & 24 & 6,2 & 416,9 & 4,9 & 14 & 3,6 & 218,0 & 2,6 \\
\hline De 20,1 a 50 & 39 & 10,1 & $1.199,7$ & 14,2 & 35 & 9,1 & $1.238,6$ & 14,6 & 15 & 3,9 & 476,7 & 5,6 \\
\hline De 50,1 a 100 & 4 & 1,0 & 259,4 & 3,0 & 30 & 7,8 & $1.325,6$ & 15,7 & 5 & 1,3 & 327,2 & 3,8 \\
\hline Más de 100 & - & - & - & - & 4 & 1,0 & 750,4 & 8,9 & - & - & - & - \\
\hline Totales & 247 & 64,5 & $3.609,1$ & 42,7 & 101 & 26,3 & $3.806,3$ & 45,0 & 35 & 9,1 & $1.030,9$ & 12,3 \\
\hline $\begin{array}{l}\text { Totales de } \\
\text { arroceros y } \\
\text { hectáreas en } \\
\text { las islas }\end{array}$ & 423 & 46,7 & $6.799,4$ & 30,3 & 337 & 37,2 & $11.357,5$ & 50,6 & 145 & 16,1 & $4.289,1$ & 19,1 \\
\hline
\end{tabular}

NOTAS 1 Y 2: Los \% están hallados sobre el total de los socios valencianos y tierras en su poder en el total de los tres sindicatos.

FUENTE: F.S.A.A.E. Delegación Zona Sur. Sevilla.

ELABORACION: Propia. 
de 10,1 a 50-, que hace posible una posición bastante acomodada; y una gran propiedad, que supra las $50 \mathrm{y}$, sobre todo, las 100 hectáreas, los valencianos consiguieron situarse, en su mayoría, en esa mediana propiedad -como se observa en el cuadro 10-, quedando afincados en estas tierras. Durante todo el lapso de tiempo, más de la mitad de los socios quedan incluidos en este grupo, y van a reunir otro tanto de las tierras; pero además, se observa que a medida que pasan los años las cifras se incrementa. Lo contrario ocurre con los pequeños propietarios, lo que nos ratifica en lo expuesto más arriba, a saber: que aquellos que no consiguen un cierto bienestar se marchan. En la base de la serie queda patente el incremento de la gran propiedad, sobre todo entre los que tienen entre 50 y 100 hectáreas, de ahí que actualmente buena parte del arrozal sevillano esté en manos de valencianos, bien individualmente, bien formando sociedades.

A la misma conclusión se llega al analizar la estrucura de la propiedad valenciana (cuadros 11 y 12), donde de nuevo se comprueba el predominio de los sindicatos de Sevilla y Villafranco en socios y tierras, y cómo se nota una cierta concentración de la propiedad en el paso de los diez años que separan a 1965 de 1975, sobre todo en el Sindicato de Sevilla.

De este rápido balance se llega a una evidencia notable y ya detectada al analizar otros aspectos: el papel primordial y fundamental que han jugado y siguen jugando los valencianos en los arrozales de las Marismas del Guadalqui$v^{2}{ }^{2}$. Por otra parte, hay que dejar igualmente subrayado que a medida que ha ido pasando el tiempo cada vez se han ido sumando más andaluces a la empresa arrocera, y actualmente podemos afirmar, sin miedo a equivocamos, que se la reparten a partes prácticamente iguales unos y otros.

2. Para una visión más amplia del fenómeno, véase González Arteaga, J. Las Marismas del Guadalquivir: etapas de su aprovechamiento económico. Edit. C.P. "Antonio Cuevas" (Puebla del Río). Sevilla, 1993. 


\section{ANEXO}

VALENCIANOS EN LAS ISLAS DEL GUADALQUIVIR. 1955

Poblado Sexo Edad Naturaleza

Ocupación

Años de

residencia

\begin{tabular}{|c|c|c|c|c|}
\hline Rincón & V & 58 & Sueca & Agricultor \\
\hline & M & 57 & Sueca & $\mathrm{S} / \mathrm{L}$ \\
\hline & V & 40 & Alberique & Campo \\
\hline & V & 29 & Valencia & Chófer \\
\hline & $\mathrm{M}$ & 29 & Valencia & $\mathrm{S} / \mathrm{L}$ \\
\hline & V & 32 & Almusafes & Campo \\
\hline & M & 30 & Almusafes & $\mathrm{S} / \mathrm{L}$ \\
\hline & $\mathrm{V}$ & 39 & Sueva & Agricultor \\
\hline & M & 24 & Egipto & $\mathrm{S} / \mathrm{L}$ \\
\hline & V & 55 & Sueca & Agricultor \\
\hline & M & 26 & Sueca & $\mathrm{S} / \mathrm{L}$ \\
\hline & $\mathrm{M}$ & 23 & Sueca & $\mathrm{S} / \mathrm{L}$ \\
\hline & M & 21 & Sueca & $\mathrm{S} / \mathrm{L}$ \\
\hline & M & 44 & Fortaleny & $\mathrm{S} / \mathrm{L}$ \\
\hline & V & 33 & Almusafes & Campo \\
\hline & M & 30 & Almusafes & $\mathrm{S} / \mathrm{L}$ \\
\hline & V & 11 & Almusafes & - \\
\hline & V & 42 & Almusafes & Campo \\
\hline & M & 36 & Almusafes & $\mathrm{S} / \mathrm{L}$ \\
\hline & V & 12 & Almusafes & Colegio \\
\hline & M & 11 & Almusafes & Colegio \\
\hline & V & 50 & Sueca & Agricultor \\
\hline & M & - & Sueca & $\mathrm{S} / \mathrm{L}$ \\
\hline & V & 36 & Valencia & Campo \\
\hline & M & 36 & Valencia & $\mathrm{S} / \mathrm{L}$ \\
\hline Alfonso XIII & V & 31 & Almusafes & Campo \\
\hline & M & 28 & Almusafes & $\mathrm{S} / \mathrm{L}$ \\
\hline & $\mathrm{V}$ & 3 & Almusafes & - \\
\hline & V & 42 & Sueca & Campo \\
\hline & M & 45 & Sueca & $\mathrm{S} / \mathrm{L}$ \\
\hline & V & 20 & Sueca & Estudiante \\
\hline & $\mathrm{M}$ & 13 & Sueca & Escolar \\
\hline
\end{tabular}




\begin{tabular}{|c|c|c|c|c|c|}
\hline Poblado & Sexo & Edad & Naturaleza & Ocupación & $\begin{array}{l}\text { Años de } \\
\text { residencia }\end{array}$ \\
\hline & V & 43 & Sueca & Campo & 8 \\
\hline & M & 38 & Sueca & $\mathrm{S} / \mathrm{L}$ & 8 \\
\hline & M & 13 & Sueca & Escolar & 8 \\
\hline & V & 55 & Sueca & Campo & 2 \\
\hline & M & 56 & Sueca & $\mathrm{S} / \mathrm{L}$ & 2 \\
\hline & V & 25 & Sueca & Campo & 2 \\
\hline & M & 19 & Sueca & $\mathrm{S} / \mathrm{L}$ & 2 \\
\hline & V & 64 & Fortaleny & Agricultor & 1 \\
\hline & M & 58 & Fortaleny & $\mathrm{S} / \mathrm{L}$ & 1 \\
\hline & V & 27 & Fortaleny & Agricultor & 1 \\
\hline & V & 17 & Fortaleny & Agricultor & 1 \\
\hline & V & 50 & Almusafe & Agricultor & 8 \\
\hline & V & 49 & Sueca & Agricultor & 10 \\
\hline & V & 31 & Sueca & Panadero & 6 \\
\hline & M & 35 & Sueca & $\mathrm{S} / \mathrm{L}$ & 6 \\
\hline & V & 7 & Sueca & Escolar & 6 \\
\hline & V & 5 & Sueca & Escolar & 5 \\
\hline & M & 67 & Sueca & $\mathrm{S} / \mathrm{L}$ & 6 \\
\hline & V & 34 & El Palmar & Agricultor & 8 \\
\hline & M & 32 & El Palmar & $\mathrm{S} / \mathrm{L}$ & 8 \\
\hline & M & 12 & El Palmar & Colegio & 8 \\
\hline & M & 6 & El Palmar & Colegio & 6 \\
\hline & V & 40 & Cofrente & Campo & 9 \\
\hline & V & 60 & Sueca & Campo & 6 \\
\hline \multirow[t]{12}{*}{ El Puntal } & V & 57 & Valencia & Campo & 9 \\
\hline & M & 51 & Valencia & $\mathrm{S} / \mathrm{L}$ & 9 \\
\hline & V & 20 & Valencia & Campo & 9 \\
\hline & V & 37 & Sueca & Industrial & 4 \\
\hline & M & 26 & Sueca & $\mathrm{S} / \mathrm{L}$ & 4 \\
\hline & V & 71 & Sueca & Industrial & 2 \\
\hline & V & 57 & Sueca & Campo & 8 \\
\hline & M & 51 & Sueca & $\mathrm{S} / \mathrm{L}$ & 8 \\
\hline & V & 25 & Sueca & Campo & 8 \\
\hline & V & 22 & Sueca & Campo & 8 \\
\hline & M & 14 & Sueca & $\mathrm{S} / \mathrm{L}$ & 8 \\
\hline & V & 25 & El Palmar & Campo & 6 \\
\hline
\end{tabular}


Poblado Sexo Edad Naturaleza

Ocupación

Años de

residencia

$\begin{array}{lrlll}\text { M } & 45 & \text { Pinedo } & \text { S/L } & 6 \\ \text { V } & 20 & \text { El Palmar } & \text { Campo } & 6 \\ \text { V } & 15 & \text { El Palmar } & \text { Escolar } & 6 \\ \text { M } & 14 & \text { El Palmar } & \text { S/L } & 6 \\ \text { V } & 56 & \text { Pulimar del Juan } & \text { Campo } & 8 \\ \text { M } & 48 & \text { Sueca } & \text { S/L } & 8 \\ \text { V } & 24 & \text { Sueca } & \text { Campo } & 8 \\ \text { M } & 16 & \text { Sueca } & \text { Escolar } & 8 \\ \text { M } & 12 & \text { Sueca } & \text { Escolar } & 8 \\ \text { M } & 10 & \text { Sueca } & \text { Escolar } & 8 \\ \text { V } & 36 & \text { Sueca } & \text { Campo } & 6 \\ \text { V } & 3 & \text { Valencia } & \text { Escolar } & 3 \\ \text { V } & 30 & \text { Alginet } & \text { Canpo } & 3 \\ \text { M } & 27 & \text { Alginet } & \text { S/L } & 3 \\ \text { V } & 40 & \text { Catarroja } & \text { Campo } & 6 \\ \text { M } & 35 & \text { Catarroja } & \text { S/L } & 6 \\ \text { M } & 14 & \text { Catarroja } & \text { Escolar } & 6 \\ \text { V } & 49 & \text { Masanava } & \text { Campo } & 4 \\ \text { M } & 45 & \text { Játiva } & \text { S/L } & 4 \\ \text { V } & 15 & \text { Játiva } & \text { Escolar } & 4 \\ \text { M } & 45 & \text { Real de Moratín } & \text { S/L } & 8 \\ \text { V } & 28 & \text { Sueca } & \text { Empleado } & 1 \\ \text { M } & 23 & \text { Sueca } & \text { S/L } & 1 \\ \text { V } & 34 & \text { Sueca } & \text { Labador1 } & \end{array}$

V 34 Sueca

Labrador 1

M $27 \quad$ Sueca

S/L

M 3 Sueca

V 41 Masalabés

-

M $38 \quad$ Masalabés

Labrador

V 13 Masalabés

S/L

M 11 Masalabés

Colegio

\section{8 Masalabés}

Colegio

Sueca

Colegio

$\mathrm{S} / \mathrm{L}$

Sueca

Campo

Sueca

Campo

Sueca

Campo 


\begin{tabular}{|c|c|c|c|c|c|}
\hline Poblado & Sexo & Edad & Naturaleza & Ocupación & $\begin{array}{l}\text { Años de } \\
\text { residencia }\end{array}$ \\
\hline & M & 35 & Carcagente & S/L & 4 \\
\hline & M & 6 & Carcagente & Escolar & 4 \\
\hline & V & 32 & Sueca & Jornalero & 3 \\
\hline & $\mathrm{M}$ & 30 & Sueca & $\mathrm{S} / \mathrm{L}$ & 3 \\
\hline & $\mathrm{V}$ & 3 & Sueca & - & 3 \\
\hline & $\mathrm{M}$ & 56 & Gandía & S/L & 6 \\
\hline & $\mathrm{M}$ & 35 & Benifalló & $\mathrm{S} / \mathrm{L}$ & 6 \\
\hline & V & 30 & Benifalló & Campo & 6 \\
\hline & V & 56 & Sueca & Jornalero & 4 \\
\hline & $\mathrm{M}$ & 54 & Sueca & $\mathrm{S} / \mathrm{L}$ & 4 \\
\hline & M & 27 & Sueca & $\mathrm{S} / \mathrm{L}$ & 4 \\
\hline & $\mathrm{M}$ & 26 & Sueca & $\mathrm{S} / \mathrm{L}$ & 4 \\
\hline & V & 39 & Sueca & Labrador & 4 \\
\hline & M & 38 & Sueca & $\mathrm{S} / \mathrm{L}$ & 4 \\
\hline & M & 14 & Sueca & Escolar & 4 \\
\hline & M & 12 & Sueca & Escolar & 4 \\
\hline & V & 9 & Sueca & Escolar & 4 \\
\hline & M & 3 & Sueca & - & 3 \\
\hline & V & 43 & Villagozal & Agricultor & 6 \\
\hline & M & 36 & Villagozal & $\mathrm{S} / \mathrm{L}$ & 6 \\
\hline & $\mathrm{M}$ & 13 & Villagozal & $\mathrm{S} / \mathrm{L}$ & 6 \\
\hline & V & 10 & Villagozal & Escolar & 6 \\
\hline & M & 64 & Villagozal & $\mathrm{S} / \mathrm{L}$ & 6 \\
\hline & V & 40 & Sueca & Labrador & 2 \\
\hline & M & 38 & Sueca & $\mathrm{S} / \mathrm{L}$ & 2 \\
\hline & V & 15 & Sueca & Escolar & 2 \\
\hline & V & 12 & Sueca & Escolar & 2 \\
\hline & V & 9 & Sueca & Escolar & 2 \\
\hline & V & 2 & Sueca & - & 2 \\
\hline & $\mathrm{M}$ & 70 & Sueca & $\mathrm{S} / \mathrm{L}$ & 2 \\
\hline & V & 46 & Sueca & Labrador & 2 \\
\hline & $\mathrm{M}$ & 42 & Sueca & $\mathrm{S} / \mathrm{L}$ & 2 \\
\hline & M & 15 & Sueca & $\mathrm{S} / \mathrm{L}$ & 2 \\
\hline & V & 35 & Sueca & Agricultor & 4 \\
\hline & M & 32 & Sueca & $\mathrm{S} / \mathrm{L}$ & 4 \\
\hline & V & 8 & Sueca & Escolar & 4 \\
\hline
\end{tabular}




\begin{tabular}{|c|c|c|c|c|c|}
\hline Poblado & Sexo & Edad & Naturaleza & Ocupación & $\begin{array}{l}\text { Años de } \\
\text { residencia }\end{array}$ \\
\hline & M & 5 & Sueca & Escolar & 4 \\
\hline & $\mathrm{M}$ & 46 & Sueca & $\mathrm{S} / \mathrm{L}$ & 4 \\
\hline & $\mathrm{V}$ & 27 & Sueca & Labrador & 4 \\
\hline & V & 49 & Sueca & Agricultor & 4 \\
\hline & M & 46 & Sueca & $\mathrm{S} / \mathrm{L}$ & 4 \\
\hline & V & 20 & Sueca & Agricultor & 4 \\
\hline & M & 15 & Sueca & $\mathrm{S} / \mathrm{L}$ & 4 \\
\hline & M & 4 & Sueca & Escolar & 4 \\
\hline & V & 44 & Sueca & Agricultor & 8 \\
\hline & M & 40 & Sueca & $\mathrm{S} / \mathrm{L}$ & 8 \\
\hline & V & 16 & Sueca & Campo & 8 \\
\hline & M & 15 & Sueca & $\mathrm{S} / \mathrm{L}$ & 8 \\
\hline & V & 41 & Sueca & Agricultor & 4 \\
\hline & M & 40 & Sueca & $\mathrm{S} / \mathrm{L}$ & 4 \\
\hline & V & 12 & Sueca & Escolar & 4 \\
\hline & $\mathrm{V}$ & 9 & Sueca & Escolar & 4 \\
\hline & M & 3 & Sueca & - & 3 \\
\hline & V & 28 & Sueca & Agricultor & 2 \\
\hline & M & 22 & Sueca & $\mathrm{S} / \mathrm{L}$ & 2 \\
\hline & V & 55 & Sueca & - & - \\
\hline & V & 54 & Sueca & Agricultor & 3 \\
\hline & M & 50 & Sueca & $\mathrm{S} / \mathrm{L}$ & 3 \\
\hline & V & 24 & Sueca & Campo & 3 \\
\hline & M & 20 & Sueca & $\mathrm{S} / \mathrm{L}$ & 3 \\
\hline & M & 18 & Sueca & $\mathrm{S} / \mathrm{L}$ & 3 \\
\hline . & V & 30 & Játiva & Industrial & 4 \\
\hline & M & 23 & Catarтоја & $\mathrm{S} / \mathrm{L}$ & 4 \\
\hline & M & 55 & Sagunto & $\mathrm{S} / \mathrm{L}$ & - \\
\hline & V & 50 & Sueca & Agricultor & 4 \\
\hline & M & 46 & Sueca & $\mathrm{S} / \mathrm{L}$ & 4 \\
\hline & V & 46 & Sueca & Campo & 4 \\
\hline & V & 23 & Sueca & Campo & 4 \\
\hline & M & 20 & Sueca & $\mathrm{S} / \mathrm{L}$ & 4 \\
\hline & V & 18 & Sueca & Campo & 4 \\
\hline & V & 11 & Sueca & Colegio & 4 \\
\hline & V & 56 & Sueca & Agricultor & 6 \\
\hline
\end{tabular}




\begin{tabular}{|c|c|c|c|c|c|}
\hline Poblado & Sexo & Edad & Naturaleza & Ocupación & $\begin{array}{l}\text { Años de } \\
\text { residencia }\end{array}$ \\
\hline & M & 50 & Sueca & $\mathrm{S} / \mathrm{L}$ & 6 \\
\hline & V & 23 & Sueca & Campo & 6 \\
\hline & V & 20 & Sueca & Campo & 6 \\
\hline & V & 19 & Sueca & Estudiante & 6 \\
\hline & V & 15 & Sueca & Estudiante & 6 \\
\hline & V & 9 & Sueca & Escolar & 6 \\
\hline & M & 8 & Sueca & Escolar & 6 \\
\hline & V & 30 & Sueca & Agricultor & 2 \\
\hline & M & 31 & Sueca & $\mathrm{S} / \mathrm{L}$ & 2 \\
\hline & V & 45 & Valencia & Agricultor & 3 \\
\hline & M & 40 & Valencia & $\mathrm{S} / \mathrm{L}$ & 3 \\
\hline & V & 50 & Sueca & Agricultor & 14 \\
\hline & M & 45 & Sueca & $\mathrm{S} / \mathrm{L}$ & 14 \\
\hline & V & 21 & Sueca & Campo & 14 \\
\hline & V & 14 & Sueca & Campo & 14 \\
\hline & V & 45 & Sueca & Agricultor & 10 \\
\hline & M & 43 & Sueca & $\mathrm{S} / \mathrm{L}$ & 10 \\
\hline & V & 31 & Sueca & Campo & 10 \\
\hline & M & 15 & Sueca & $\mathrm{S} / \mathrm{L}$ & 10 \\
\hline & V & 8 & Sueca & Escolar & 8 \\
\hline & V & 35 & Sueca & Campo & 1 \\
\hline & V & 42 & Villa Gozalo & Agricultor & 2 \\
\hline & M & 36 & Villa Gozalo & $\mathrm{S} / \mathrm{L}$ & 2 \\
\hline & V & 54 & Cullera & Agricultor & 4 \\
\hline & M & 39 & Cullera & $\mathrm{S} / \mathrm{L}$ & 4 \\
\hline & V & 25 & Cullera & Labrador & 4 \\
\hline & M & 20 & Cullera & $\mathrm{S} / \mathrm{L}$ & 4 \\
\hline & M & 43 & Sueca & $\mathrm{S} / \mathrm{L}$ & 6 \\
\hline & V & 37 & Murcia & Labrador & 6 \\
\hline & V & 9 & Sueca & Escolar & 6 \\
\hline & V & 4 & Sueca & - & 4 \\
\hline & V & 53 & Venibeciles & Labrador & 2 \\
\hline & M & 50 & Venibeciles & $\mathrm{S} / \mathrm{L}$ & 2 \\
\hline & V & 24 & Venibeciles & Labrador & 2 \\
\hline & $\mathrm{V}$ & 18 & Venibeciles & Labrador & 2 \\
\hline & M & 15 & Venibeciles & Escolar & 2 \\
\hline
\end{tabular}




\begin{tabular}{|c|c|c|c|c|c|}
\hline Poblado & Sexo & Edad & Naturaleza & Ocupación & $\begin{array}{l}\text { Años de } \\
\text { residencia }\end{array}$ \\
\hline & M & 11 & Venibeciles & Escolar & 2 \\
\hline & V & 48 & Valencia & Labrador & 5 \\
\hline & M & 46 & Valencia & $\mathrm{S} / \mathrm{L}$ & 5 \\
\hline & M & 13 & Valencia & Escolar & 5 \\
\hline & $\mathrm{V}$ & 46 & Alcira & Agricultor & 20 \\
\hline & M & 39 & Carcagente & $\mathrm{S} / \mathrm{L}$ & 20 \\
\hline & V & 12 & Carcagente & Escolar & 12 \\
\hline & V & 10 & Carcagente & Escolar & 10 \\
\hline & M & 5 & Carcagente & Escolar & 5 \\
\hline & V & 56 & Sueca & Labrador & 2 \\
\hline & M & 56 & Sueca & $\mathrm{S} / \mathrm{L}$ & 2 \\
\hline & V & 27 & Sueca & Labrador & 4 \\
\hline & M & 36 & Sueca & $\mathrm{S} / \mathrm{L}$ & 4 \\
\hline & M & 55 & Carcagente & $\mathrm{S} / \mathrm{L}$ & 4 \\
\hline & V & 59 & Carcagente & Campo & 14 \\
\hline & V & 18 & Carcagente & Campo & 14 \\
\hline & V & 55 & Almusafes & Campo & 14 \\
\hline & M & 51 & Almusafes & $\mathrm{S} / \mathrm{L}$ & 6 \\
\hline & M & 26 & Almusafes & $\mathrm{S} / \mathrm{L}$ & 6 \\
\hline & M & 11 & Almusafes & Escolar & 6 \\
\hline & V & 42 & Sueca & Campo & 3 \\
\hline & M & 38 & Sueca & $\mathrm{S} / \mathrm{L}$ & 3 \\
\hline \multirow[t]{14}{*}{ Isla Menor } & V & 40 & Favasete & Jornalero & 2 \\
\hline & M & 38 & Favasete & $\mathrm{S} / \mathrm{L}$ & 2 \\
\hline & V & 10 & Favasete & Escolar & 2 \\
\hline & $\mathrm{V}$ & 7 & Favasete & Escolar & 2 \\
\hline & M & 5 & Favasete & Escolar & 2 \\
\hline & M & 1 & Isla Menor & - & 1 \\
\hline & M & 30 & Valencia & $\mathrm{S} / \mathrm{L}$ & 10 \\
\hline & V & 42 & Farreta & Agricultor & 2 \\
\hline & V & 42 & Sueca & Labrador & 2 \\
\hline & M & 40 & Sueca & $\mathrm{S} / \mathrm{L}$ & 2 \\
\hline & $\mathrm{V}$ & 19 & Sueca & Labrador & 2 \\
\hline & V & 14 & Sueca & Escolar & 2 \\
\hline & M & 12 & Sueca & Escolar & 2 \\
\hline & M & 9 & Sueca & Escolar & 2 \\
\hline
\end{tabular}




\begin{tabular}{lrrllc}
\hline Poblado & Sexo Edad & Naturaleza & Ocupación & $\begin{array}{c}\text { Años de } \\
\text { residencia }\end{array}$ \\
\hline & M & 9 & Sueca & Escolar & 2 \\
& V & 40 & Sueca & Labrador & 2 \\
& M & 38 & Sueca & S/L & 2 \\
& V & 16 & Sueca & Labrador & 2 \\
& V & 7 & Sueca & Escolar & 2 \\
Isla Mínima & V & 40 & Sueca & Industrial & 3 \\
& M & 42 & Sueca & S/L & 3 \\
& V & 42 & Sueca & Campo & 3 \\
& V & 9 & Sueca & Escolar & 3 \\
V & 6 & Sueca & Escolar & 3 \\
V & 40 & Sueca & Industrial & 6 \\
M & 43 & Sueca & S/L & 6 \\
M & 17 & Sueca & Estudiante & 6 \\
M & 8 & Sueca & Escolar & 6 \\
V & 5 & Sueca & Escolar & 5 \\
V & 4 & Sueca & Escolar & 4
\end{tabular}

FUENTE: Archivo Municipal de Puebla del Río. Padrón de 1955.

ELABORACIÓN: Propia. 